\title{
Looking for Myocardial Viability After a STICH Trial: Not Enough to Close the Door
}

I function due to coronary artery disease (CAD) is steadily increasing as a consequence of the aging of the population and of improved survival of patients with acute coronary syndromes and currently represents the first cause of heart failure (1). Mechanisms of LV dysfunction in CAD patients are complex and have been elucidated in recent years. A landmark understanding was that ischemic LV dysfunction may be sustained by repetitive ischemia, myocardial stunning, and hibernation and, therefore, completely or partially reversible in a substantial number of patients who undergo revascularization $(2,3)$. From this evidence emerged the concept of viability or viable myocardium-that is, the distinction between reversible and irreversible dysfunction due to myocardial necrosis - that became part of the diagnostic workup of patients with ischemic LV dysfunction. Accordingly, several imaging approaches looking at myocardial viability were developed with the aim of selecting patients in whom recovery of LV function (mostly defined as increase of ejection fraction $[\mathrm{EF}]$ at rest) and improvement of prognosis would outweigh the risk of surgical revascularization. From these studies, it was also evident that most patients with ischemic LV dysfunction show evidence of viable myocardium as identified by viability testing $(4,5)$.

Received Dec. 20, 2011; revision accepted Jan. 10, 2012.

For correspondence or reprints contact: Pasquale Perrone-Filardi, Department of Internal Medicine, Cardiovascular Sciences and Immunology, Federico

II University, Via Pansini, 5, I-80131 Naples, Italy.

E-mail: fpperron@unina.it

Published online Feb. 9, 2012.

COPYRIGHT (c) 2012 by the Society of Nuclear Medicine, Inc.

DOI: 10.2967/jnumed.111.102210
Most studies for which the aim was to identify viable myocardium were designed having as the endpoint the shortterm increase of regional or global LV function at rest after revascularization, both of which were assumed to represent the surrogate endpoint for clinical benefits-that is, survival and qualityof-life improvement. The evidence from these studies clearly indicated that methods that explore the integrity of cell membrane or metabolic activity, such as nuclear techniques, are more sensitive for predicting recovery of $\mathrm{LV}$ function than those that explore the contractile reserve of dysfunctional myocardium (6-8). Additionally, it was also demonstrated that revascularization of viable myocardium influences recovery of LV remodeling independently of changes in EF (9), pointing to additional mechanisms of clinical benefits.

\section{VIABILITY AND CLINICAL OUTCOMES IN PATIENTS WITH ISCHEMIC LV DYSFUNCTION}

Until the Surgical Treatment for Ischemic Heart Failure (STICH) viability study (10), only 1 randomized study (11) investigated whether evaluation of viability using PET to select patients with LV dysfunction for surgical revascularization or medical therapy would reduce the composite endpoint of death, myocardial infarction, and hospitalization for cardiac causes at $1 \mathrm{y}$. That study did not demonstrate a significant reduction of events in PET-assisted patients, compared with controls, but the results were mainly explained by the large number of patients in whom therapeutic management was not consistent with the indication of PET. In fact, a statistically significant benefit was observed in a post hoc analysis in patients in whom PET recommendations were followed.

A previous metaanalysis (12) investigated the impact of revascularization in relation to the evidence of viability in patients with LV dysfunction. That metaanalysis reported a mortality rate of 3,088 patients with mean EF of $32 \%$ followed for 25 mo who underwent nuclear (either PET or SPECT) or dobutamine echocardiography for the assessment of viability before revascularization. In that analysis, patients with viability who remained on medical therapy showed the worst survival rate, whereas in patients with viability who underwent revascularization, a $79.6 \%$ reduction in annual mortality rate (from $16 \%$ to $3.2 \%$ ) was observed. Interestingly, the benefit of revascularization was independent of the technique used to assess viability, and lack of viability was associated with no differences in outcome between medical therapy and revascularization. The impact of that metaanalysis was quite relevant and supported the clinical need of viability testing in patients with LV dysfunction to avoid useless and risky surgical revascularization and to provide clinical benefit to selected patients. Several limitations were appropriately underscored by the authors of the metaanalysis, including potential selection and publication bias, the observational and not randomized design of the studies, presence of comorbidities, heterogeneity of the studies collected in the analysis, and lack of optimization of medical therapy. Yet the conclusions of that metaanalysis were endorsed by the European Society of Cardiology and the European Association for CardioThoracic Surgery, whose myocardial revascularization recommended the evaluation of viability to assist management of patients with LV ischemic dysfunction (13). The STICH viability trial (10) did not confirm an impact of viability on outcome of patients under- 
going revascularization or medical therapy, cautioning against the relevance of viability in the management of patients with LV dysfunction. Yet, although the STICH trial represents a remarkable study exploring a relevant hypothesis, some aspects should be carefully analyzed when interpreting the results.

\section{STICH TRIAL}

In the main STICH study (14), for which 1,212 patients with ischemic LV dysfunction and an EF of $35 \%$ or less were enrolled, no significant all-cause mortality differences were observed by intention-to-treat analysis between patients undergoing coronary artery bypass grafting (CABG), compared with optimal medical therapy. In the $\mathrm{CABG}$ group, however, the prespecified secondary endpoint of cardiovascular deaththat is, the mortality outcome more likely to be influenced by $\mathrm{CABG}$ was reduced by $19 \%$, with borderline statistical significance $(P=0.05)$, whereas the composite endpoint of cardiac death and hospitalization for cardiac cause was significantly reduced by $26 \%$. Interestingly, a significant $30 \%$ all-cause mortality reduction was observed in the CABG group in the as-treated analysis, which took into account the substantial crossover rate (17\%) of patients assigned to medical therapy who underwent $\mathrm{CABG}$, pointing to the possibility that such crossover may have diluted the benefit of $\mathrm{CABG}$ in the primary intention-totreat analysis. Therefore, as acknowledged by the authors, the results of the STICH study do not definitively deny the advantage of CABG in LV dysfunction but rather provide, from secondary analysis, provisional evidence in favor of revascularization in ischemic LV dysfunction.

In the same issue of the New England Journal of Medicine as that in which the main STICH study was reported (14), Bonow et al. (10) reported the results of the viability STICH substudy that assessed, in about half the population randomized in the main study, the influence of viability on clinical outcome in patients assigned to medical therapy or CABG. The results of that study showed that survival of patients with viability was significantly longer than that of patients without viability, but patients with viable myocardium undergoing CABG did not show survival benefit, compared with those treated with optimized medical therapy. The study results thus disputed the conclusion of the previous metaanalysis (12) and questioned the recommendations of guidelines for viability-guided treatment in patients with ischemic LV dysfunction (10).

\section{IS VIABLE MYOCARDIUM NO LONGER ALIVE AFTER STICH?}

The STICH trial was originally designed to test the influence of viability, evaluated with SPECT, on all-cause mortality in patients undergoing CABG or optimized medical therapy. Yet, viability assessment significantly hampered recruitment of patients, and the protocol was subsequently amended to make viability testing optional and, at the choice of investigators, performed by either SPECT or dobutamine echocardiography. This change substantially influenced the enrollment of patients in the substudy, yielding a final population of 601 , compared with the 1,212 patients of the main trial. This difference in populations resulted in a comparison of 4 subgroups of patients with and without viability who were undergoing medical therapy or surgery but who were not numerically balanced because most $(81 \%)$ showed viable myocardium, raising concerns about the statistical adequacy of the method to identify differences among groups.

Even more relevant is the definition of viability using SPECT adopted in the study. Patients with viable myocardium were those showing at least 11 (of 17) myocardial segments defined as viable on the basis of regional tracer uptake using rest-redistribution, rest-stress, or rest-redistribution-reinjection SPECT protocols. However, this criterion was arbitrarily defined and not validated in clinical trials. In fact, the power of SPECT using thresholds of relative tracer uptake to predict recovery of regional function may be not high, because myocardial dysfunctional segments may not recover after revascularization despite preserved tracer uptake (6-8). In addition, it was not specified whether the 11 or more segments required for defining viability were counted only among those showing contractile dysfunction at rest, as would have been appropriate, or among all myocardial segments, and no data were reported on the degree of contractile dysfunction (hypokinesia, akinesia, or dyskinesia) of viable segments. It is obvious that although myocardial segments with normal function at rest and preserved tracer uptake represent viable myocardium, they cannot contribute to recovery of function after revascularization and, therefore, should be excluded from the sum of viable segments. Moreover, the criterion of relative tracer uptake using SPECT could be misleading in patients with severe LV dysfunction and extensive vessel involvement in whom no truly normally perfused myocardium may be present, thus leading to overestimation of viability by semiquantitative analysis (15). In these patients, PET assessment of viability using absolute flow values would likely be more accurate.

Although in the STICH trial dobutamine echocardiography and SPECT were interchangeably used for viability evaluation, these techniques show different accuracies for predicting recovery of regional function or of EF after revascularization $(6-8)$ and a combination of echocardiographic and SPECT findings is more accurate than either technique alone (16). Yet, no such analysis was reported for STICH patients. In addition, the percentage of patients defined as viable by SPECT or dobutamine echocardiography should have been presumably different, because the 2 techniques are often not concordant in the same dysfunctional territories $(6,17)$. Although the authors reported that no influence could be found regarding the type of test used, the limited number of patients, most of them undergoing SPECT, likely prevented the exclusion of any influence of the type of test on the results. In fact, using the definitions of viability adopted in the study, and because of the prevalence of SPECT testing, only 114 (19\%) of 601 patients were identified as not showing viable 
myocardium. This percentage of viable patients would have been presumably much lower had dobutamine echocardiography been used more often or had segments with preserved tracer uptake but normal function not been counted $(6,17)$.

In addition, no information on regional and global LV function or volume changes after treatment was reporteda missed opportunity that should foster additional analysis. In fact, an analysis of $\mathrm{EF}$ and $\mathrm{LV}$ volume changes after treatment and of the influence of these changes on clinical outcomes would have substantially contributed to a better understanding of the results, because it is well known that the presence of viable myocardium favorably affects $\mathrm{LV}$ remodeling and function, which, in turn, correlate with clinical outcomes. Thus, an examination of $\mathrm{EF}$ and $\mathrm{LV}$ volume changes after treatment would have contributed to the verification of the adequacy of viability definition in the trial and its impact on clinical outcome. The fact that both revascularization and medical therapy favorably influence LV EF and remodeling (18) may have obscured the impact of viability on prognosis comparing CABG with optimized medical therapy and may explain the association between viability and longer survival observed in the study. This consideration appears to be supported by the comparable mortality rate of CABG and medically treated patients with viable myocardium - a rate substantially lower than that reported for patients without viability. Patients with viable myocardium showed significantly more preserved LV function and volumes at enrollment than did patients without viable myocardium, making the association between viability and survival no longer significant when adjusted for covariates. However, a significant favorable association between viability and the composite endpoint of cardiovascular mortality and hospitalization still remained after adjustment for baseline differences, raising the hypothesis that CABG and optimal medical therapy may provide comparable benefits in patients with viable myocardium. This hypothesis is further supported by the striking difference in mortality rate observed in the STICH study in patients with viable myocardium undergoing medical therapy ( $\sim 7 \%$ per year), compared with medically treated patients reported in the previous metaanalysis (higher than $15 \%$ per year), whereas the mortality of patients with viable myocardium undergoing $\mathrm{CABG}$ was similar to that previously reported $(\sim 6 \%$ per year) (12). Altogether, these data do not detract from the pathophysiologic and clinical relevance of viability but rather underlie the favorable effects of evidence-based medical therapy in patients with LV dysfunction and viable myocardium.

\section{PERSPECTIVES}

The assessment of myocardial viability in the selection of patients for myocardial revascularization has suffered a significant blow with the results of the STICH trial, and these results certainly represented a wakeup call for the cardiology community. However, when put in perspective and properly dissected, the STICH trial should be seen not as a definitive and final answer to the question of viability assessment but rather as a matter of reflection, particularly in the following 2 respects: proper patient selection and proper methods and criteria of assessment of viability.

The STICH trial also showed how difficult it is to perform certain clinical studies, despite the relevance of the underlying clinical question, raising the big issue of identifying new and better surrogates of disease (in the current case of myocardial viability). It certainly does not close the door on the big question of myocardial viability assessment before myocardial revascularization but will surely influence the development of future potential clinical trials in this area. In particular, advanced cardiac imaging using PET and MRI needs to be tested in this context. Thus, it is hoped that the search for new and more accurate diagnostic methods will continue, with the goal of having better and more refined criteria (19).

\section{ACKNOWLEDGMENT}

No potential conflict of interest relevant to this article was reported.

\section{Pasquale Perrone-Filardi}

Department of Internal Medicine, Cardiovascular Sciences and Immunology, Federico II University, Naples, Italy

\section{Fausto J. Pinto}

Department of Cardiology,

Lisbon University Medical School,

Lisbon, Portugal

\section{REFERENCES}

1. Ezekowitz JA, Kaul P, Bakal JA, Armstrong PW, Welsh RC, McAlister FA. Declining in-hospital mortality and increasing heart failure incidence in elderly patients with first myocardial infarction. J Am Coll Cardiol. 2009;53:13-20.

2. Schinkel AF, Bax JJ, Delgado V, Poldermans D, Rahimtoola SH. Clinical relevance of hibernating myocardium in ischemic left ventricular dysfunction. Am J Med. 2010;123:978-986.

3. Elefteriades JA, Tolis G Jr, Levi E, Mills LK, Zaret BL. Coronary artery bypass grafting in severe left ventricular dysfunction: excellent survival with improved ejection fraction and functional state. $J \mathrm{Am}$ Coll Cardiol. 1993;22:1411-1417.

4. Cleland JG, Pennell DJ, Ray SG, et al. Carvedilol hibernating reversible ischaemia trial: marker of success investigators. Myocardial viability as a determinant of the ejection fraction response to carvedilol in patients with heart failure (CHRISTMAS trial): randomised controlled trial. Lancet. 2003;362:14-21.

5. Perrone-Filardi P, Pace L, Prastaro M, et al. Dobutamine echocardiography predicts improvement of hypoperfused dysfunctional myocardium following revascularization in patients with coronary artery disease. Circulation. 1995;91:2556-2565.

6. Perrone-Filardi P, Pace L, Prastaro M, et al. Assessment of myocardial viability in patients with chronic coronary artery disease: rest- 4 hour- 24 hour ${ }^{201}$ thallium tomography vs dobutamine echocardiography. Circulation. 1996;94:2712-2719.

7. Underwood SR, Bax JJ, von Dahl J, et al. Imaging techniques for the assessment of myocardial hibernation: Report of a Study Group of the European Society of Cardiology. Eur Heart J. 2004;25:815-836.

8. Camici PG, Prasad SK, Rimoldi OE. Stunning, hibernation, and assessment of myocardial viability. Circulation. 2008;117:103-114.

9. Carluccio E, Biagioli P, Alunni G, et al. Patients with hibernating myocardium show altered left ventricular volumes and shape, which revert after revascularization: evidence that dyssynergy might directly induce cardiac remodeling. $\mathrm{J} \mathrm{Am}$ Coll Cardiol. 2006;47:969-977.

10. Bonow RO, Maurer G, Lee KL, et al. Myocardial viability and survival in ischemic left ventricular dysfunction. N Engl J Med. 2011;364:1617-1625.

11. Beanlands RS, Nichol G, Huszti E, et al. F-18-fluorodeoxyglucose positron emission tomography imaging-assisted management of patients with severe left ventricular dysfunction and suspected coronary disease: a randomized, controlled trial (PARR-2). J Am Coll Cardiol. 2007;50:2002-2012.

12. Allman KC, Shaw LJ, Hachamovitch R, Udelson JE. Myocardial viability testing and impact of revascular- 
ization on prognosis in patients with coronary artery disease and left ventricular dysfunction: a meta-analysis. J Am Coll Cardiol. 2002;39:1151-1158.

13. European Association for Percutaneous Cardiovascular Interventions, Wijns W, Kolh P, et al. Guidelines on myocardial revascularization: The Task Force on Myocardial Revascularization of the European Society of Cardiology (ESC) and the European Association for Cardio-Thoracic Surgery (EACTS). Eur Heart J. 2010;31:2501-2555.

14. Velazquez EJ, Lee KL, Deja MA, et al. Coronaryartery bypass surgery in patients with left ventricular dysfunction. N Engl J Med. 2011;364:1607-1616.
15. Pace L, Perrone Filardi P, Mainenti PP, et al. Identification of viable myocardium in patients with chronic coronary artery disease using rest-redistribution thallium-201 tomography: optimal image analysis. J Nucl Med. 1998;39:1869-1874.

16. Pace L, Perrone Filardi P, Mainenti PP, et al. Combined evaluation of rest-redistribution thallium-201 tomography and low-dose dobutamine echocardiography enhances the identification of viable myocardium in patients with chronic coronary artery disease. Eur J Nucl Med. 1998;25:744-750.

17. Panza JA, Dilsizian V, Laurienzo JM, Curiel RV, Katsiyiannis PT. Relation between thallium uptake and contractile response to dobutamine: implications regarding myocardial viability in patients with chronic coronary artery disease and left ventricular dysfunction. Circulation. 1995;91:990-998.

18. Bello D, Shah DJ, Farah GM, et al. Gadolinium cardiovascular magnetic resonance predicts reversible myocardial dysfunction and remodeling in patients with heart failure undergoing betablocker therapy. Circulation. 2003;108:19451953.

19. Pinto FJ. Myocardial viability: the search for a perfect method is not over yet. Eur Heart J. 2000;21:1039-1040. 